\title{
The Role of Immune Checkpoint Inhibitors in Leptomeningeal Disease: A Systematic Review
}

\author{
PAOLO PALMISCIANO ${ }^{1}$, ALI S. HAIDER ${ }^{2}$, CHIBUEZE D. NWAGWU ${ }^{3}$, \\ WASEEM WAHOOD ${ }^{4}$, KENNY YU $^{5}$, CHIBAWANYE I. ENE ${ }^{6}$, BARBARA J. O'BRIEN ${ }^{7}$, \\ SALAH G. AOUN ${ }^{8}$, AARON A. COHEN-GADOL ${ }^{9}$ and TAREK Y. EL AHMADIEH ${ }^{8}$ \\ ${ }^{1}$ Department of Neurosurgery, Trauma Center, Gamma Knife Center, Cannizzaro Hospital, Catania, Italy; \\ ${ }^{2}$ Texas A\&M University College of Medicine, Houston, TX, U.S.A.; \\ ${ }^{3}$ Emory University School of Medicine, Atlanta, GA, U.S.A.; \\ ${ }^{4}$ Dr. Kiran C. Patel College of Allopathic Medicine, Nova Southeastern University, Davie, FL, U.S.A.; \\ ${ }^{5}$ Department of Neurosurgical Surgery, Memorial Sloan Kettering Cancer Center, New York, NY, U.S.A.; \\ ${ }^{6}$ Department of Neurosurgery, MD Anderson Cancer Center, Houston, TX, U.S.A.; \\ ${ }^{7}$ Department of Neuro-Oncology, MD Anderson Cancer Center, Houston, TX, U.S.A.; \\ ${ }^{8}$ Department of Neurological Surgery, University of Texas Southwestern Medical Center, Dallas, TX, U.S.A.; \\ ${ }^{9}$ Department of Neurological Surgery, Indiana University School of Medicine, Indianapolis, IN, U.S.A.
}

\begin{abstract}
Background/Aim: Leptomeningeal disease (LMD) is a debilitating complication of advanced malignancies. Immunecheckpoint inhibitors (ICIs) may alter disease course. We analyzed the role and toxicity of ICIs in LMD. Materials and Methods: We systematically reviewed the literature reporting on outcome data of patients with LMD treated with ICIs. Results: We included 14 studies encompassing 61 patients. Lung-cancer (44.3\%), breast-cancer (27.9\%), and melanoma (23.0\%) were the most frequent primary tumors. Median duration of ICItreatment was 7-months (range=0.5-58.0): pembrolizumab (49.2\%), nivolumab (32.8\%), ipilimumab (18.0\%). Radiological responses included complete response (33.3\%), partial response (12.5\%), stable disease (33.3\%), progressive disease (20.8\%). Twenty-two patients developed ICI-related adverse-events, mild $(100 \%)$ andlor severe (15.6\%). Median progression-free and overall survival were 5.1 and 6.3 months, and 12-month survival was $32.1 \%$. Survival correlated with ICI agents
\end{abstract}

This article is freely accessible online.

Correspondence to: Tarek Y. El Ahmadieh, MD, Department of Neurological Surgery, University of Texas Southwestern Medical Center, 5323 Harry Hines Blvd, Dallas, TX 75390, U.S.A. Tel: +1 (312)2211336, e-mail: telahmadieh@gmail.com

Key Words: Brain metastasis, corticosteroids, immune checkpoint inhibitors, immunotherapy, leptomeningeal disease, review. $(p=0.042)$, but not with primary tumors $(p=0.144)$. Patients receiving concurrent steroids showed worse survival $(p=0.040)$. Conclusion: ICI therapy is well-tolerated in patients with LMD, but concurrent steroids may worsen survival.

Leptomeningeal disease (LMD) occurs in 5-10\% of oncological patients, most commonly from primary melanoma, lung, and breast neoplasms (1). LMD presents with new neurological impairments and focal/diffuse meningealenhancement at T1-contrast MRI, and is confirmed at cerebrospinal fluid (CSF) cytology (2). Available treatments include systemic/intrathecal chemotherapy, whole-brain/focal radiotherapy, and supportive-care; more recently, targetedtherapy and immunotherapy have been considered (3). The therapeutic goal remains palliation, with mean survival of 3-6 months $(4,5)$.

The immune system plays a major role in shaping the systemic response in neuro-oncology (6). Cancerous cells may elude the immune response by expressing immunecheckpoint ligands, such as cytotoxic T-lymphocyteassociated antigen 4 (CTLA-4) and programmed deathligand 1 (PD-L1), which suppress T-cell activation and proliferation (7). Immune-checkpoint inhibitors (ICIs) are novel agents directed at inhibiting these signals, enhancing immune recognition of tumors and T-cell response. ICIs have proven effective in brain metastases from melanoma, lung, and breast cancers, and clinical interest is expanding toward LMD (7-11). 
Although ICIs represent an exciting development in neuro-oncology, their potential role in LMD remains unclear, with data derived only from few single-institution studies. We systematically reviewed the literature to summarize the use of ICIs in LMD, analyzing their role in improving quality-of-life and survival, and their toxicity profiles.

\section{Materials and Methods}

Literature search. A systematic review was performed upon the preferred reporting items for systematic reviews and meta-analyses (PRISMA) guidelines (12). PubMed, EMBASE, Scopus, and Cochrane were searched from inception to March 23, 2021, operating the Boolean full-text search [(leptomeningeal disease OR leptomeningeal metastases OR leptomeningeal carcinomatosis OR carcinomatous meningitis OR neoplastic meningitis) AND (immunotherapy OR immune checkpoint inhibitors)]. Collected studies were exported to Mendeley; duplicates were removed. A second search was conducted on clinicaltrials.gov to identify ongoing clinical trials.

Study selection. Predetermined inclusion and exclusion criteria were set. Articles were included if they: 1) were retrospective/prospective studies including $\geq 1$ patient diagnosed with LMD and treated with ICIs; 3 ) had available data on treatment outcomes and survival; 4) were written in English. Studies were excluded if they: 1) were reviews, animal, or laboratory studies; 2) lacked data on clinical/radiological responses or survival.

Two authors independently screened titles and abstracts of all identified papers and reviewed full texts of articles that met the inclusion criteria. A third author settled any disagreements. Eligible papers were included, and references screened to identify additional articles.

Data extraction. One reviewer extracted data from each article, then confirmed independently by two additional reviewers. Data included: author, study design, age, gender, primary tumor, brain metastases and treatment, PD-L1 expression, time-interval between primary tumor and LMD, LMD symptoms and radiological appearances, CSF-cytology, ICIs and duration of treatment, additional LMD treatment, Eastern Cooperative Oncology Group performance status (ECOG-PS) pre- and post-ICIs, LMD radiological response, LMD recurrence, progression-free survival (PFS), overall survival (OS), and survival status $(13,14)$.

LMD clinical - ECOG-PS post-ICI - and radiological responses were evaluated within the first 6-months of ICIs, as suggested in the iRANO statement (15). Radiological responses were assessed based on the RANO criteria modified for LMD, describing changes in the pathological brain and spine $\mathrm{T} 1$-contrast enhancement from baseline: complete-response (CR; no evidence of disease), partialresponse (PR; $>50 \%$ decrease in the summed product of orthogonal diameters of composite measurable nodules), stable-disease (SD; no change), progressive-disease (PD; $>25 \%$ increase in the summed product of orthogonal diameters of composite measurable nodules or new sites of disease) (14).

Data synthesis and quality assessment. The primary outcomes of interest were patients' clinical outcomes, radiological responses, and survival. ICI-related adverse-events were also evaluated. Level of evidence was assessed using the 2011 Oxford Centre For Evidence-
Based Medicine guidelines (16). Meta-analysis was precluded because most included studies had levels IV-V of evidence, and hazard-ratios could not be deducted. Risk of bias was independently appraised by two reviewers applying the Joanna Briggs Institute checklists for casereports, case-series, and randomized controlled trials (17-19).

Statistical analysis. SPSS V.25 (IBM Corp, Armonk, New York) was used for statistical analysis. Continuous variables are presented as medians or means and ranges, categorical variables as frequencies and percentages. A two-sample weighted $t$-test was performed to assess differences between pre- and post-ICI ECOGPS scores. Time-intervals between the start of ICI and LMD recurrence (PFS-curve) or death (OS-curve) were estimated using the Kaplan-Meier method; the survival analyses were conducted with the log-rank test. Two-sided $p$-value $<0.05$ was considered significant for all analyses.

\section{Results}

Study selection and overview. The search yielded 289 citations, of which 14 were included in the qualitative synthesis (Supplementary File 1). Nine were case-reports, four were retrospective case-series (including 32 patients), and one was a single-arm phase-II trial (including 20 patients); levels of evidence were V, IV, and IIb, respectively (Supplementary File 2) (8-11, 20-29). Critical appraisal resulted in low risk of bias for all included articles (Supplementary File 3).

Demographics, clinical and diagnostic characteristics. A total of 61 patients were analyzed (Supplementary File 2). Median age was 57 years with female predominance (63.9\%) (Table I). Primary tumors were mostly lung-cancer $(44.3 \%)$, breast-cancer $(27.9 \%)$, and melanoma (23.0\%). Thirty-seven patients $(80.4 \%)$ presented with brain metastases, of which $31(83.8 \%)$ treated with radiotherapy: $27(73.0 \%)$ wholebrain radiotherapy (WBRT), four (10.8\%) stereotactic radiosurgery (SRS). PD-L1 expression rates were evaluated from pathological specimens of nine patients with lungcancer: high (expressed in $\geq 50 \%$ of tumor cells) in eight cases $(88.9 \%)$, low $(<50 \%)$ in one $(11.1 \%)$.

Median time-interval between primary tumor and LMD was 10 months (range $=0-48$ months). The most common LMD symptoms were headache (42.1\%), nausea/vomiting $(23.7 \%)$, diplopia $(23.7 \%)$, and facial-nerve palsy (10.5\%). LMD diagnosis was obtained with MRI T1-contrast exams and/or CSF-cytology. Neuroimaging abnormalities included diffuse leptomeningeal-enhancement of the brain (96.7\%) and/or spinal cord (3.2\%), and simultaneous lumbar-nodular disease in two patients $(3.2 \%)$. In two cases, normal MRI findings were reported (3.2\%). CSF-cytology was obtained in 37 patients: positive in $32(86.5 \%)$, negative in five $(13.5 \%)$.

Management strategies, clinical and radiological responses, and adverse events. All 61 patients received ICIs for a median of seven months (range=0.5-58.0 months) (Table II). 
The following agents were administered: pembrolizumab (Anti-PD1 monoclonal-antibody; Merck \& Co., Kenilworth, NJ, USA) $2 \mathrm{mg} / \mathrm{kg}$ q3 weeks (49.2\%); nivolumab (Anti-PD1 monoclonal-antibody; Bristol Myers Squibb, New York, NY, USA) $3 \mathrm{mg} / \mathrm{kg}$ q2 weeks (32.8\%); ipilimumab (Anti-CTLA4 monoclonal-antibody; Bristol Myers Squibb, New York, NY, USA) $3 \mathrm{mg} / \mathrm{kg}$ q3 weeks (18.0\%). Twenty-eight patients underwent concomitant treatments: WBRT $(51.1 \%)$ and SRS (3.6\%), corticosteroids (35.7\%), dabrafenib/trametinib (BRAF-inhibitor/MEK-inhibitor; Novartis, Basel, Switzerland) (10.7\%), systemic chemotherapy (3.6\%) and intrathecal methotrexate $(3.6 \%)$.

Median ECOG-PS scores were one both pre- and postICIs, and weighted means were not significantly different $(p=0.176)$. Radiological responses were assessed in 24 patients: CR in eight (33.3\%), PR in three (12.6\%), SD in eight (33.3\%), and PD in five (20.8\%).

ICI-related adverse-events were described in 22/32 patients $(68.7 \%)$ and reported using the "Common Terminology Criteria for Adverse Events, v5.0" (30). The most common were: grade-1, vomiting (27.3\%), hyperglycemia (27.3\%), constipation (22.7\%) and fatigue (22.7\%); grade-2, nausea and colitis (both 9.1\%); grade-3, headache (13.6\%).

Survival outcomes and analysis. LMD recurrence rate was $60.5 \%$, and death occurred in 34 patients $(55.7 \%)$. Median PFS and OS were 5.1 and 6.3 months, respectively (Table II). The 6-month PFS and OS rates were $46.1 \%$ and $50.9 \%$, whereas the 12 -month rates were $28.2 \%$ and $32.1 \%$, respectively. The 6-month and 12-month survival rates stratified for primary tumor were $51.8 \%$ and $29.6 \%$ for lungcancer, $30.0 \%$ and $10.0 \%$ for breast-cancer, $57.1 \%$ and $42.8 \%$ for melanoma, with median OS of $7.0,5.2$, and 9.1 months, respectively. The 6-month and 12-month survival rates stratified for ICIs were $50.0 \%$ and $40.9 \%$ for pembrolizumab, $55.0 \%$ and $25.0 \%$ for nivolumab, $45.4 \%$ and $27.3 \%$ for ipilimumab, with median OS of 5.9, 7.0, and 4.1 months, respectively. Median OS in patients receiving and not-receiving steroids concurrent to ICIs were 1.9 and 6.1 months, respectively.

No statistical difference in PFS was found based on the primary tumor $(p=0.131)$, ICI agents $(p=0.095)$, or concurrent use of steroids $(p=0.92)$. No statistical difference in OS was found based on the primary tumor $(p=0.144)$ (Figure 1), but OS was significantly lower in patients with concurrent use of steroids $(p=0.040)$. Significant difference in OS was found based on ICI agents $(p=0.042)$. Subgroup analyses showed significantly higher OS comparing pembrolizumab (median OS 35.0-months) with ipilimumab (median OS 4.1 months) $(p=0.018)$ and nivolumab (median OS 33.5 months) with ipilimumab $(p=0.031)$ for patients with melanoma, but not between pembrolizumab and nivolumab for patients with melanoma $(p=0.808)$ and lung
Table I. Summary of demographics, clinical, and diagnostic characteristics of all pooled patients with leptomeningeal disease.

\begin{tabular}{|c|c|}
\hline $\begin{array}{l}\text { Characteristics } \\
\text { (no. of patients for whom } \\
\text { information is available) }\end{array}$ & $\begin{array}{l}\text { Value (among } \\
\text { patients with } \\
\text { available data) }\end{array}$ \\
\hline Cohort size (no.) & 61 \\
\hline \multicolumn{2}{|l|}{ Demographics } \\
\hline Median age, range (years) $(n=41)$ & $57(33-93)$ \\
\hline Gender (female) $(n=50)$ & $39(63.9 \%)$ \\
\hline Primary tumor $(n=61)$ & No. $(\%)$ \\
\hline Lung & $27(44.3 \%)$ \\
\hline Breast & $17(27.9 \%)$ \\
\hline Melanoma & $14(23.0 \%)$ \\
\hline Kidney & $1(1.6 \%)$ \\
\hline Head \& neck squamous cell carcinoma & $1(1.6 \%)$ \\
\hline Ovary & $1(1.6 \%)$ \\
\hline Secondary metastases $(n=46)$ & No. $(\%)$ \\
\hline Brain & $37(80.4 \%)$ \\
\hline Lymph nodes & $16(34.8 \%)$ \\
\hline Bone (Long bones, Spine, Skull base) & $13(28.3 \%)$ \\
\hline Lung & $6(13.0 \%)$ \\
\hline Liver & $4(8.7 \%)$ \\
\hline Treatment brain metastases $(n=37)$ & No. $(\%)$ \\
\hline Radiation therapy & $31(83.8 \%)$ \\
\hline Chemotherapy & $5(13.5 \%)$ \\
\hline Surgery & $4(10.8 \%)$ \\
\hline Intrathecal methotrexate & $1(2.7 \%)$ \\
\hline PD-L1 expression $(n=9)$ & No. $(\%)$ \\
\hline High expression & $8(88.9 \%)$ \\
\hline Low expression & $1(11.1 \%)$ \\
\hline $\begin{array}{l}\text { Time between primary tumor } \\
\text { and LMD, months (range) }\end{array}$ & $10.0(0-48.0)$ \\
\hline \multicolumn{2}{|l|}{ LMD presenting symptoms $(\mathrm{n}=38)$} \\
\hline \multicolumn{2}{|l|}{ No. $(\%)$} \\
\hline Headache & $16(42.1 \%)$ \\
\hline Nausea/Vomit & $9(23.7 \%)$ \\
\hline Diplopia & $9(23.7 \%)$ \\
\hline Cranial nerve VII palsy & $4(10.5 \%)$ \\
\hline Others & $18(47.4 \%)$ \\
\hline \multicolumn{2}{|c|}{ Leptomeninges T1-contrast enhancement $(n=61)$} \\
\hline \multicolumn{2}{|l|}{ No. $(\%)$} \\
\hline Brain diffuse & $59(96.7 \%)$ \\
\hline Spine diffuse & $2(3.2 \%)$ \\
\hline Lumbar nodule & $2(3.2 \%)$ \\
\hline No contrast & $2(3.2 \%)$ \\
\hline CSF cytology $(n=37)$ & No. $(\%)$ \\
\hline Positive & $32(86.5 \%)$ \\
\hline Negative & $5(13.5 \%)$ \\
\hline
\end{tabular}

LMD: Leptomeningeal disease; CSF: cerebrospinal fluid.

cancer (pembrolizumab median OS 12.5 months; nivolumab median OS 10.6 months) $(p=0.342)$.

\section{Discussion}

LMD is a devastating complication of advanced systemic malignancies, with uncertain optimal management and 
Table II. Summary of leptomeningeal disease treatment strategies and outcomes of all pooled patients.

\begin{tabular}{lc}
\hline Characteristics & Value (among \\
patients with \\
(no. of patients for whom & available data) \\
information is available) & 61 \\
\hline Cohort size (no.) & \\
& \\
ICI therapy (n=61) & \\
No. (\%) & $30(49.2 \%)$ \\
Pembrolizumab & $20(32.8 \%)$ \\
Nivolumab & $11(18.0 \%)$ \\
Ipilimumab & $18(66.6 \%)$ \\
Lung cancer (n=27) & $9(33.3 \%)$ \\
Nivolumab & \\
Pembrolizumab & $11(78.6 \%)$ \\
Melanoma (n=14) & $2(14.3 \%)$ \\
Ipilimumab & $1(7.1 \%)$ \\
Pembrolizumab & \\
Nivolumab & $17(100 \%)$ \\
Breast (n=17) & \\
Pembrolizumab & $1(100 \%)$ \\
Renal cell carcinoma (n=1) & \\
Nivolumab & \\
Head and neck squamous cell carcinoma (n=1) & \\
Pembrolizumab & $12(68.7 \%)$ \\
Ovarian cancer (n=1) & \\
Pembrolizumab & \\
Duration of ICI therapy (months), \\
median (range) (n=41)
\end{tabular}

dismal survival $(1,2)$. This systematic review examined the role of ICIs in patients with LMD, noting their positive impact on quality-of-life and survival, coupled with lowrates of severe adverse-events.

The most common primary tumors were lung cancer, breast cancer, and melanoma, reflecting their overall prevalence among the general oncological population. These neoplasms represent the main oncological targets against which current ICIs have been proven effective, further confirming the

\begin{tabular}{lc}
$\begin{array}{l}\text { Characteristics } \\
\text { (no. of patients for whom } \\
\text { information is available) }\end{array}$ & $\begin{array}{c}\text { Value (among } \\
\text { patients with } \\
\text { available data) }\end{array}$ \\
\hline Type \& Grade of ICI-related adverse & \\
events (CTCAE v5.0) (n=22) & \\
No. (\%) & \\
G1: Vomiting & $6(27.3 \%)$ \\
Hyperglycemia & $6(27.3 \%)$ \\
Constipation & $5(22.7 \%)$ \\
Fatigue & $5(22.7 \%)$ \\
Diarrhea & $2(9.1 \%)$ \\
Rash & $2(9.1 \%)$ \\
G2: Nausea & $2(9.1 \%)$ \\
Colitis & $2(9.1 \%)$ \\
Pneumonitis & $1(4.5 \%)$ \\
G3: Headache & $3(13.6 \%)$ \\
Bone pain & $1(4.5 \%)$ \\
Lymphocytes/Platelet count decreased & $1(4.5 \%)$ \\
Muscle weakness lower limbs & $1(4.5 \%)$ \\
Inflammatory arthritis & $1(4.5 \%)$ \\
Strongyloides infection & $1(4.5 \%)$ \\
Recurrence of LMD (n=43) & $26(60.5 \%)$ \\
Outcome (months) & \\
Progression-free survival (n=39) & Median (range) \\
Overall survival (n=53) & $5.1(0.2-39.0)$ \\
Status (n=61) & $6.3(0.5-58.7)$ \\
Alive & No. $(\%)$ \\
Dead & $27(44.3 \%)$ \\
\hline
\end{tabular}

ICI: Immune checkpoint inhibitor; LMD: leptomeningeal disease; CTCAE: Common Terminology Criteria for Adverse Events; ECOG: Eastern Cooperative Oncology Group.

promising role of ICIs in simultaneously modulating immunologic microenvironments of primary tumors and metastatic lesions $(7,31)$. The median time-interval from primary tumor to LMD was 10 months, largely lower than the 2-4 years range reported in previous series, probably because most patients presented with systemic metastases at the initial diagnosis $(32,33)$. In patients with brain metastases, resection and adjuvant radiotherapy of the surgical cavity have been demonstrated to increase risks of LMD by allowing tumor-cell spilling into the CSF $(34,35)$. In contrast, most of our pooled patients underwent radiotherapy alone for brain metastases preceding LMD. Our findings may corroborate a different pathological LMD mechanism, suggesting the direct transmeningeal and intra-subarachnoid space spreading of cancer cells from adjacent parenchymal brain metastases, not adequately prevented by radiation (35).

Clinical evaluation with T1-contrast MRI and CSFcytology assist the diagnostic workflow. Symptoms arise from the leptomeningeal dissemination of tumor cells, leading to CSF-flow obstruction with increased intracranial 

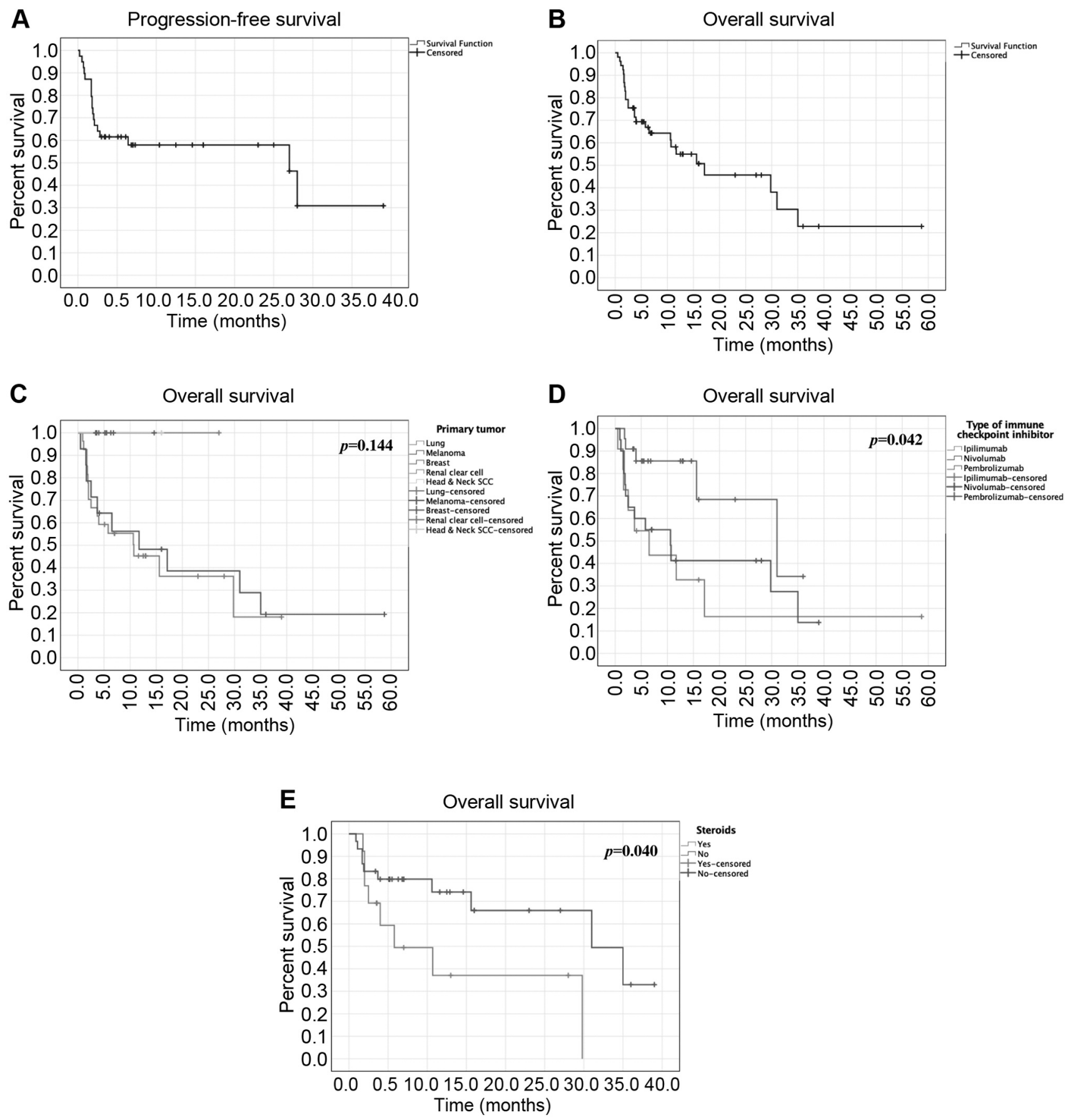

Figure 1. Kaplan-Meier survival curves of patients with available individual data: A. Progression-free survival ( $n=39)$ and B. Overall survival $(n=53)$ of the total pooled cohort; $C$. Overall survival $(n=53)$ based on primary tumor; D. Overall survival ( $n=53)$ based on immune checkpoint inhibitor therapy; E. Overall survival ( $n=43)$ based on the use of steroids concurrent to immune checkpoint inhibitor use.

pressure, and meningeal invasion (36). Accordingly, most of our pooled patients presented with headache, vomiting, and cranial-nerve palsies, but available data on hydrocephalus were scarce. In two singular cases, memory loss and auditory hallucinations were ascribed to the presence of temporoinsular LMD lesions $(8,21)$. Most LMD diagnoses were obtained after new symptom onset or upon routine followup surveillance in patients with metastatic disease. Most 
patients exhibited diffuse brain and/or spinal cord MRI contrast-enhancement and confirmatory positive CSFcytology. As estimated in up to $25 \%$ cases, we found few symptomatic patients presenting with normal MRI and positive-CSF cytology (3.2\%), or negative CSF-cytology and pathological MRI (13.5\%) (1). Hence, the clinical context remains key and needs to be integrated with imaging and CSF cytology for diagnosing LMD.

Challenges in the management of LMD relate to the poor clinical conditions of patients with systemic malignancies, with palliative therapies mostly intended to improve quality of life while avoiding toxicity. In our cohort, radiotherapy and corticosteroids were the most frequent treatments administered in concomitance with ICIs. Their preferred role derives from their ability to shrink tumors and facilitate CSFflow, thus relieving symptoms and improving the distribution of intrathecal chemotherapeutics, which act against tumor cells within the CSF $(37,38)$. SRS is a feasible option in focal nodular-LMD, and, when adopted preoperatively, may also reduce the surgical risk of CSF cancerous spread from brain metastases $(34,39)$. However, no patients were deemed eligible for these SRS protocols across the collected studies. Despite showing promising results in LMD, moleculartherapies selectively treat neoplasms expressing specific target mutations, failing to be implemented in routine clinical practice (40). Indeed, only three of our pooled patients with melanoma $(3 / 14,21 \%)$ were eligible to receive concomitant BRAK/MEK inhibitors, corroborating the limits of targeted therapies and suggesting the superior feasibility of immunotherapeutic strategies.

Cancer cells may express immune-checkpoint molecules capable of suppressing protective T-cell responses; this has been also demonstrated for brain metastases $(6,31)$. ICIs promote anti-tumoral immune-modulatory responses by selectively preventing the interaction between these immunesuppressive molecules and cytotoxic T-cells in both primary and metastatic cancer sites. Several clinical-trials demonstrated the role of ICIs in controlling systemic and intracranial metastases from melanoma, lung, and breast malignancies (41-43). The immune response can also access the CSF compartment; thus ICIs may simultaneously treat primary tumors and leptomeningeal metastases, leading to the rapid lesion shrinkage with improvement of neurological symptoms and cognitive functioning $(7,9)$. Based on primary tumor origin, three ICIs/monoclonal antibodies have been validated for the treatment of LMD: ipilimumab acting against CTLA4; nivolumab and pembrolizumab targeting the PD-1/PD-L1 axis $(9,10,28)$. In our cohort, ICIs correlated with prompt symptomatic relief and long-lasting positive functional status. In addition, at post-treatment radiological assessment, rates of stable disease $(33.3 \%)$ and radiological responses (partial 12.6\%; complete $33.3 \%$ ) were higher than progression rates $(20.9 \%)$. Due to LMD aggressive nature, coupled with the poor life expectancy of patients with systemic malignancies, treatments should primarily aim at preserving performance statuses and relieving debilitating symptoms. As opposed to the described limits of other therapies, ICIs appear to effectively prolong favorable functional status by contrasting LMD clinical and radiological progression.

In this review, the median OS (6.3 months), the 6-month $(50.9 \%)$, and the 12 -month survival rates $(32.1 \%)$ were superior to those reported in previous large series of LMD (median OS 3 months; 6-month and 12-month survival rates of $28 \%$ and $10 \%$, respectively) $(44,45)$. In those series, the extensive heterogeneity in primary tumors and treatment strategies accounted for the poor therapeutic response and survival. In contrast, the patients included in our analysis were selected based on their primary tumors for which ICIs had already proven to be effective. However, our findings were similar to survival obtained from previous studies selectively analyzing the role of radiotherapy or targetedtherapies in $\operatorname{LMD}(46,47)$. In LMD, ICIs may improve survival on par with the other recommended treatments, but the poor baseline clinical status of affected patients likely limits their therapeutic efficacy.

Previous series described better survival of patients with LMD from breast cancer compared to melanoma and lung cancers, with approximate median OS of 4.5, 4.0, and 3.1 months respectively $(4,5,28)$. In our cohort, we found superior median OS in patients with LMD from melanoma (9.1 months) compared to lung (7.0 months) and breast (5.2 months) cancers, but differences were not significant. Our findings may reflect the superior activity of single-agent checkpoint blockade in primary and metastatic melanomas and/or lung-cancers when compared to breast-cancers, which demonstrate better responses only with multiple ICI agents or multimodal adjuvant treatments (48-50). We noted significant differences in survival based on ICIs, regardless of primary tumors. Nivolumab yielded optimal median OS (7.0 months) and 6-month survival $(55.0 \%)$, but pembrolizumab showed higher 12-month survival $(40.9 \%)$. These differences were likely attributed to the large heterogeneity in tumor origins. Recent clinical-trials demonstrated that pembrolizumab and nivolumab significantly prolonged OS in patients with advanced melanoma when compared with ipilimumab (51, 52). Similarly, our subgroup analysis returned statistically higher OS in pembrolizumab and nivolumab treatments over ipilimumab for LMD originating from melanoma. We also found no significant difference in OS between nivolumab and pembrolizumab treatments for melanoma and lung cancers, as per previous studies describing their interchangeable role in the treatment of these neoplasms (53-55). Our findings likely relate to the suggested connections between immune microenvironments of primary tumors and LMD. They further support the feasibility of extending the clinical 
applicability of ICIs, currently available mostly for primary tumors, to treat concomitant LMD.

As described in experimental models, steroids significantly reduce circulating T-cells and increase PD-1 expression, hampering anti-tumoral immune responses and ICIs' efficacy $(56,57)$. Steroids showed a detrimental effect on the survival of patients with brain metastases undergoing ICI therapy (58, 59). Brastianos et al. (9) reported worse OS in patients with LMD receiving steroids concurrent to pembrolizumab (2.4 months) compared to the non-steroid group (5.1 months), but differences were not significant $(p=0.32)$. In our study, we found a significant correlation between OS and steroids use $(p=0.040)$, with median OS of 1.9 and 6.1 months for steroid and non-steroid groups, respectively. ICIs and steroids independently provide symptomatic relief in patients with LMD, but their combination may negatively affect survival. Their deleterious impact on survival may be also connected to the likely worse burden of disease in patients who receive steroids for palliation. Our findings may suggest avoiding steroids in patients undergoing ICI therapy for LMD, but further prospective studies are required to better define their combined role, especially in patients with significant disease burden.

Patients with LMD are often considered too frail for aggressive treatments, and toxicity profiles should be evaluated first. In our cohort, we found a $68.7 \%$ rate of ICIrelated adverse events, mostly mild, self-limited and with a low-impact on quality of life. Contrarily, mild radiotherapy adverse events (hair loss and skin reactions) occur in approximately $100 \%$ of patients, posing a significant cosmetic burden and affecting quality of life $(3,37)$. Severe ICI-related adverse-events (headache, bone marrow depression) were reported in 5 patients $(15.6 \%)$ but were successfully treated with temporary ICI discontinuation and/or addition of steroids $(8,9,24)$. Of note, the use of steroids for mitigating ICI-related adverse events has not been correlated with a negative impact on survival (59). In comparison, severe radiotherapy adverse events (neuroaxis toxicity, bone marrow depression) and intrathecalchemotherapy adverse events (chemical and aseptic meningitis) often require hospitalization with surgery and/or prolonged antibiotic courses (37). Overall, rates and severities of our pooled adverse events were comparable with those of patients with primary and metastatic tumors undergoing ICIs (60). However, while recent studies reported incidences of fatal ICI-related adverse events ranging 0.3$13 \%$, ICIs caused no life threatening side effects in our cohort, also showing better toxicity profiles than radiotherapy and intrathecal-chemotherapy $(2,40,60)$.

Limitations. Our study has several limitations. Most included studies were case reports and retrospective case series, subjected to publication and selection biases. The only clinical trial was single-arm and open-label, not comparing ICIs' efficacy and toxicity profiles to other available treatments. The poor level-of-evidence of included studies precluded a metaanalysis and challenged the statistical power of this study. Some patient-level data could not be extracted in five studies, limiting our sample size and pooled analysis $(9-11,28,29)$.

\section{Conclusion}

LMD is a serious late-stage complication of systemic malignancies with dismal prognoses. ICIs appear to be safe and effective in patients with LMD, improving survival while maintaining favorable quality of life. The concurrent use of steroids correlates with worse survival. Future randomized controlled trials are required to further assess the ICIs' role in the multimodal management of LMD, analyze their combined effect with steroids, and compare their efficacy to novel targeted therapies.

\section{Supplementary Material}

Available at: https://www.dropbox.com/sh/ktmmfizdxqjs585/AA ChVN01tD10B7PVzGfHogKMa?dl=0

\section{Conflicts of Interest}

The Authors have no relevant financial or non-financial interests to disclose.

\section{Authors' Contributions}

Paolo Palmisciano: Conceptualization, Methodology, Data analysis, Writing - Original draft preparation; Ali S. Haider: Resources, Writing - Reviewing and Editing; Chibueze D. Nwagwu: Resources, Writing - Reviewing and Editing; Waseem Wahood: Data analysis, Writing - Reviewing and Editing; Kenny Yu: Resources, Writing Reviewing and Editing; Chibawayne I. Ene: Resources, Writing Reviewing and Editing; Barbara J. O'Brien: Resources, Writing Reviewing and Editing; Salah G. Aoun: Statistical analysis, Writing - Reviewing and Editing; Aaron A. Cohen-Gadol: Resources, Writing - Reviewing and Editing; Tarek Y. El Ahmadieh: Methodology, Resources, Writing - Reviewing and Editing, Supervision.

\section{References}

1 Nayar G, Ejikeme T, Chongsathidkiet P, Elsamadicy AA, Blackwell KL, Clarke JM, Lad SP and Fecci PE: Leptomeningeal disease: current diagnostic and therapeutic strategies. Oncotarget 8(42): 73312-73328, 2017. PMID: 29069871. DOI: $10.18632 /$ oncotarget.20272

2 Wang N, Bertalan MS and Brastianos PK: Leptomeningeal metastasis from systemic cancer: Review and update on management. Cancer 124(1): 21-35, 2018. PMID: 29165794. DOI: $10.1002 /$ cncr.30911

3 Buszek SM and Chung C: Radiotherapy in leptomeningeal disease: a systematic review of randomized and non-randomized trials. Front Oncol 9: 1224, 2019. PMID: 31803614. DOI: $10.3389 /$ fonc .2019 .01224 
4 Scott BJ, Oberheim-Bush NA and Kesari S: Leptomeningeal metastasis in breast cancer - a systematic review. Oncotarget 7(4): 3740-3747, 2016. PMID: 26543235. DOI: 10.18632/oncotarget. 5911

5 Cheng $\mathrm{H}$ and Perez-Soler R: Leptomeningeal metastases in nonsmall-cell lung cancer. Lancet Oncol 19(1): e43-e55, 2018. PMID: 29304362. DOI: 10.1016/S1470-2045(17)30689-7

6 Sampson JH, Gunn MD, Fecci PE and Ashley DM: Brain immunology and immunotherapy in brain tumours. Nat Rev Cancer 20(1): 12-25, 2020. PMID: 31806885. DOI: 10.1038/ s41568-019-0224-7

7 Aquilanti E and Brastianos PK: Immune checkpoint inhibitors for brain metastases: a primer for neurosurgeons. Neurosurgery 87(3): E281-E288, 2020. PMID: 32302389. DOI: 10.1093/ neuros/nyaa095

8 Arias Ron D, Labandeira CM, Areses Manrique MC, Sampedro Domarco P, Abdulkader I, García-Mata J, Rolfo C, GonzálezRivas D and Fírvida JL: Dramatic response of leptomeningeal carcinomatosis to nivolumab in PD-L1 highly expressive nonsmall cell lung cancer: a case report. Front Oncol 9: 819, 2019. PMID: 31552167. DOI: 10.3389/fonc.2019.00819

9 Brastianos PK, Lee EQ, Cohen JV, Tolaney SM, Lin NU, Wang N, Chukwueke U, White MD, Nayyar N, Kim A, AlvarezBreckenridge C, Krop I, Mahar MK, Bertalan MS, Shaw B, Mora JL, Goss N, Subramanian M, Nayak L, Dietrich J, Forst DA, Nahed BV, Batchelor TT, Shih HA, Gerstner ER, Moy B, Lawrence D, Giobbie-Hurder A, Carter SL, Oh K, Cahill DP and Sullivan RJ: Single-arm, open-label phase 2 trial of pembrolizumab in patients with leptomeningeal carcinomatosis. Nat Med 26(8): 1280-1284, 2020. PMID: 32483359. DOI: 10.1038/s41591-020-0918-0

10 Dudnik E, Yust-Katz S, Nechushtan H, Goldstein DA, Zer A, Flex D, Siegal $\mathrm{T}$ and Peled N: Intracranial response to nivolumab in NSCLC patients with untreated or progressing CNS metastases. Lung Cancer 98: 114-117, 2016. PMID: 27393516. DOI: 10.1016/j.lungcan.2016.05.031

11 Lavaud J, Blom A, Longvert C, Fort M, Funck-Brentano E and Saiag P: Pembrolizumab and concurrent hypo-fractionated radiotherapy for advanced non-resectable cutaneous squamous cell carcinoma. Eur J Dermatol 29(6): 636-640, 2019. PMID: 31903954. DOI: 10.1684/ejd.2019.3671

12 Page MJ, McKenzie JE, Bossuyt PM, Boutron I, Hoffmann TC, Mulrow CD, Shamseer L, Tetzlaff JM, Akl EA, Brennan SE, Chou R, Glanville J, Grimshaw JM, Hróbjartsson A, Lalu MM, Li T, Loder EW, Mayo-Wilson E, McDonald S, McGuinness LA, Stewart LA, Thomas J, Tricco AC, Welch VA, Whiting P and Moher D: The PRISMA 2020 statement: an updated guideline for reporting systematic reviews. BMJ 372: n71, 2021. PMID: 33782057. DOI: 10.1136/bmj.n71

13 Oken MM, Creech RH, Tormey DC, Horton J, Davis TE, McFadden ET and Carbone PP: Toxicity and response criteria of the Eastern Cooperative Oncology Group. Am J Clin Oncol 5(6): 649-655, 1982. PMID: 7165009.

14 Chamberlain M, Junck L, Brandsma D, Soffietti R, Rudà R, Raizer J, Boogerd W, Taillibert S, Groves MD, Le Rhun E, Walker J, van den Bent M, Wen PY and Jaeckle KA: Leptomeningeal metastases: a RANO proposal for response criteria. Neuro Oncol 19(4): 484492, 2017. PMID: 28039364. DOI: 10.1093/neuonc/now183

15 Okada H, Weller M, Huang R, Finocchiaro G, Gilbert MR, Wick W, Ellingson BM, Hashimoto N, Pollack IF, Brandes AA,
Franceschi E, Herold-Mende C, Nayak L, Panigrahy A, Pope WB, Prins R, Sampson JH, Wen PY and Reardon DA: Immunotherapy response assessment in neuro-oncology: a report of the RANO working group. Lancet Oncol 16(15): e534-e542, 2015. PMID: 26545842. DOI: 10.1016/S1470-2045(15)00088-1

16 Howick J, Chalmers I, Glasziou P, Greenhalgh T, Heneghan C, Liberati A, Moschetti I, Phillips B and Thornton H: Explanation of the 2011 Oxford Centre for Evidence-Based Medicine (OCEBM) Levels of Evidence (Background Document). Oxford Center for Evidence-Based Medicine, 2011. Available at: https://www.cebm.ox.ac.uk/resources/levels-of-evidence/ocebmlevels-of-evidence [Last accessed on April 19, 2021]

17 Joanna Briggs Institute: Checklist for Case Reports, 2020. Available at: https://jbi.global/critical-appraisal-tools [Last accessed on April 19, 2021]

18 Joanna Briggs Institute: Checklist for Case Series, 2020. Available at: https://jbi.global/critical-appraisal-tools [Last accessed on April 19, 2021]

19 Joanna Briggs Institute: Checklist for Randomized Controlled Trials, 2020. Available at: https://jbi.global/critical-appraisaltools [Last accessed on April 19, 2021]

20 Bot I, Blank CU and Brandsma D: Clinical and radiological response of leptomeningeal melanoma after whole brain radiotherapy and ipilimumab. J Neurol 259(9): 1976-1978, 2012. PMID: 22527228. DOI: 10.1007/s00415-012-6488-4

21 Gion M, Remon J, Caramella C, Soria JC and Besse B: Symptomatic leptomeningeal metastasis improvement with nivolumab in advanced non-small cell lung cancer patient. Lung Cancer 108: 72-74, 2017. PMID: 28625652. DOI: 10.1016/j.lungcan.2017.02.022

22 Bover M, Yarza R and Docampo LI: Four-year lasting sustained complete response after nivolumab in a patient with non-smallcell lung cancer and confirmed leptomeningeal carcinomatosis: changing the paradigm. Clin Lung Cancer 21(1): e1-e5, 2020. PMID: 31601524. DOI: 10.1016/j.cllc.2019.07.012

23 Bonomi L, Bettini AC, Arnoldi E, Chirco A, Ghilardi L, Manara O, Roscigno M, Da Pozzo LF and Tondini CA: Nivolumab efficacy in leptomeningeal metastasis of renal cell carcinoma: a case report. Tumori 106(6): NP76-NP78, 2020. PMID: 32041500. DOI: $10.1177 / 0300891620904411$

24 Misir Krpan A, Rakusic Z and Herceg D: Primary leptomeningeal melanomatosis successfully treated with PD-1 inhibitor pembrolizumab: A case report. Medicine (Baltimore) 99(50): e22928, 2020. PMID: 33327228. DOI: 10.1097/ MD.0000000000022928

25 Nakashima K, Demura Y, Oi M, Tabata M, Tada T, Shiozaki K, Akai $\mathrm{M}$ and Ishizuka $\mathrm{T}$ : Whole-brain radiation and pembrolizumab treatment for a non-small-cell lung cancer patient with meningeal carcinomatosis lacking driver oncogenes led to a long-term survival. Intern Med 59(11): 1433-1435, 2020. PMID: 32161221. DOI: 10.2169/internalmedicine.4232-19

26 Wu RC, Newman W, Patanowitz L, Branstetter BF, Amankulor $\mathrm{N}$ and Tarhini AA: Long-term control of leptomeningeal disease after radiation therapy and nivolumab in a metastatic melanoma patient. Immunotherapy 12(11): 763-769, 2020. PMID: 32571131. DOI: 10.2217/imt-2019-0004

27 Marinova AM, Reilly JL, Wong V, Weiss S and Olszanski AJ: Metastatic melanoma with leptomeningeal disease. J Adv Pract Oncol 12(1): 79-83, 2021. PMID: 33552663. DOI: $10.6004 /$ jadpro.2021.12.1.6 
28 Geukes Foppen MH, Brandsma D, Blank CU, van Thienen JV, Haanen JB and Boogerd W: Targeted treatment and immunotherapy in leptomeningeal metastases from melanoma. Ann Oncol 27(6): 1138-1142, 2016. PMID: 26961150. DOI: 10.1093/annonc/mdw 134

29 Hendriks LEL, Bootsma G, Mourlanette J, Henon C, Mezquita L, Ferrara R, Audigier-Valette C, Mazieres J, Lefebvre C, Duchemann B, Cousin S, le Pechoux C, Botticella A, De Ruysscher D, Dingemans AC and Besse B: Survival of patients with non-small cell lung cancer having leptomeningeal metastases treated with immune checkpoint inhibitors. Eur J Cancer 116: 182-189, 2019. PMID: 31203193. DOI: 10.1016/ j.ejca.2019.05.019

30 National Cancer Institute: Common Terminology Criteria for Adverse Events (CTCAE) v5.0., 2017. Available at: https://ctep.cancer.gov/protocoldevelopment/electronic_applicati ons/ctc.htm\#ctc_50 [Last accessed on April 19, 2021]

31 Fares J, Ulasov I, Timashev P and Lesniak MS: Emerging principles of brain immunology and immune checkpoint blockade in brain metastases. Brain 144(4): 1046-1066, 2021. PMID: 33893488. DOI: 10.1093/brain/awab012

32 Wolf A, Donahue B, Silverman JS, Chachoua A, Lee JK and Kondziolka D: Stereotactic radiosurgery for focal leptomeningeal disease in patients with brain metastases. J Neurooncol 134(1): 139-143, 2017. PMID: 28536993. DOI: 10.1007/s11060-017-2497-6

33 Ferguson SD, Bindal S, Bassett RL Jr, Haydu LE, McCutcheon IE, Heimberger AB, Li J, O’Brien BJ, Guha-Thakurta N, Tetzlaff MT, Tawbi H, Davies MA and Glitza IC: Predictors of survival in metastatic melanoma patients with leptomeningeal disease (LMD). J Neurooncol 142(3): 499-509, 2019. PMID: 30847840 DOI: $10.1007 / \mathrm{s} 11060-019-03121-2$

34 Nguyen TK, Sahgal A, Detsky J, Atenafu EG, Myrehaug S, Tseng CL, Husain Z, Heyn C, Maralani P, Ruschin M, Perry J and Soliman H: Predictors of leptomeningeal disease following hypofractionated stereotactic radiotherapy for intact and resected brain metastases. Neuro Oncol 22(1): 84-93, 2020. PMID: 31412120. DOI: 10.1093/neuonc/noz144

35 Prabhu RS, Turner BE, Asher AL, Marcrom SR, Fiveash JB, Foreman PM, Press RH, Patel KR, Curran WJ, Breen WG, Brown PD, Jethwa KR, Grills IS, Arden JD, Foster LM, Manning MA, Stern JD, Soltys SG and Burri SH: A multiinstitutional analysis of presentation and outcomes for leptomeningeal disease recurrence after surgical resection and radiosurgery for brain metastases. Neuro Oncol 21(8): 10491059, 2019. PMID: 30828727. DOI: 10.1093/neuonc/noz049

36 Pan Z, Yang G, He H, Yuan T, Wang Y, Li Y, Shi W, Gao P, Dong $\mathrm{L}$ and Zhao G: Leptomeningeal metastasis from solid tumors: clinical features and its diagnostic implication. Sci Rep 8(1): 10445, 2018. PMID: 29992998. DOI: 10.1038/s41598-01828662-w

37 Pan Z, Yang G, He H, Zhao G, Yuan T, Li Y, Shi W, Gao P, Dong $\mathrm{L}$ and $\mathrm{Li} \mathrm{Y}$ : Concurrent radiotherapy and intrathecal methotrexate for treating leptomeningeal metastasis from solid tumors with adverse prognostic factors: A prospective and single-arm study. Int J Cancer 139(8): 1864-1872, 2016. PMID: 27243238. DOI: $10.1002 / \mathrm{ijc} .30214$

38 Boogerd W, van den Bent MJ, Koehler PJ, Heimans JJ, van der Sande JJ, Aaronson NK, Hart AA, Benraadt J and Vecht ChJ: The relevance of intraventricular chemotherapy for leptomeningeal metastasis in breast cancer: a randomised study. Eur J Cancer 40(18): 2726-2733, 2004. PMID: 15571954. DOI: 10.1016/j.ejca.2004.08.012

39 Patel KR, Burri SH, Asher AL, Crocker IR, Fraser RW, Zhang C, Chen Z, Kandula S, Zhong J, Press RH, Olson JJ, Oyesiku NM, Wait SD, Curran WJ, Shu HK and Prabhu RS: Comparing preoperative with postoperative stereotactic radiosurgery for resectable brain metastases: a multi-institutional analysis. Neurosurgery 79(2): 279-285, 2016. PMID: 26528673. DOI: 10.1227/NEU.0000000000001096

40 Thomas KH and Ramirez RA: Leptomeningeal disease and the evolving role of molecular targeted therapy and immunotherapy. Ochsner J 17(4): 362-378, 2017. PMID: 29230121.

41 Goldberg SB, Gettinger SN, Mahajan A, Chiang AC, Herbst RS, Sznol M, Tsiouris AJ, Cohen J, Vortmeyer A, Jilaveanu L, Yu J, Hegde U, Speaker S, Madura M, Ralabate A, Rivera A, Rowen E, Gerrish H, Yao X, Chiang V and Kluger HM: Pembrolizumab for patients with melanoma or non-small-cell lung cancer and untreated brain metastases: early analysis of a non-randomised, open-label, phase 2 trial. Lancet Oncol 17(7): 976-983, 2016. PMID: 27267608. DOI: 10.1016/S1470-2045(16)30053-5

42 Margolin K, Ernstoff MS, Hamid O, Lawrence D, McDermott D, Puzanov I, Wolchok JD, Clark JI, Sznol M, Logan TF, Richards J, Michener T, Balogh A, Heller KN and Hodi FS: Ipilimumab in patients with melanoma and brain metastases: an open-label, phase 2 trial. Lancet Oncol 13(5): 459-465, 2012. PMID: 22456429. DOI: 10.1016/S1470-2045(12)70090-6

43 Schmid P, Adams S, Rugo HS, Schneeweiss A, Barrios CH, Iwata H, Diéras V, Hegg R, Im SA, Shaw Wright G, Henschel V, Molinero L, Chui SY, Funke R, Husain A, Winer EP, Loi S, Emens LA and IMpassion130 Trial Investigators: Atezolizumab and nab-paclitaxel in advanced triple-negative breast cancer. $\mathrm{N}$ Engl J Med 379(22): 2108-2121, 2018. PMID: 30345906. DOI: 10.1056/NEJMoa1809615

44 Brower JV, Saha S, Rosenberg SA, Hullett CR and Ian Robins $\mathrm{H}$ : Management of leptomeningeal metastases: Prognostic factors and associated outcomes. J Clin Neurosci 27: 130-137, 2016. PMID: 26778048. DOI: 10.1016/j.jocn.2015.11.012

45 Hyun JW, Jeong IH, Joung A, Cho HJ, Kim SH and Kim HJ: Leptomeningeal metastasis: Clinical experience of 519 cases. Eur J Cancer 56: 107-114, 2016. PMID: 26841095. DOI: 10.1016/j.ejca.2015.12.021

46 Gong L, Xiong M, Huang Z, Miao L and Fan Y: Icotinib might be effective for the treatment of leptomeningeal carcinomatosis in non-small cell lung cancer with sensitive EGFR mutations. Lung Cancer 89(3): 268-273, 2015. PMID: 26141219. DOI: 10.1016/j.lungcan.2015.06.001

47 Ozdemir Y, Yildirim BA and Topkan E: Whole brain radiotherapy in management of non-small-cell lung carcinoma associated leptomeningeal carcinomatosis: evaluation of prognostic factors. J Neurooncol 129(2): 329-335, 2016. PMID: 27306442. DOI: $10.1007 / \mathrm{s} 11060-016-2179-9$

48 Mahoney KM, Freeman GJ and McDermott DF: The next immune-checkpoint inhibitors: PD-1/PD-L1 blockade in melanoma. Clin Ther 37(4): 764-782, 2015. PMID: 25823918. DOI: $10.1016 /$ j.clinthera.2015.02.018

49 Assi HI, Kamphorst AO, Moukalled NM and Ramalingam SS: Immune checkpoint inhibitors in advanced non-small cell lung cancer. Cancer 124(2): 248-261, 2018. PMID: 29211297. DOI: $10.1002 /$ encr.31105 
50 Polk A, Svane IM, Andersson M and Nielsen D: Checkpoint inhibitors in breast cancer - Current status. Cancer Treat Rev 63: 122-134, 2018. PMID: 29287242. DOI: 10.1016/j.ctrv.2017.12.008

51 Robert C, Schachter J, Long GV, Arance A, Grob JJ, Mortier L, Daud A, Carlino MS, McNeil C, Lotem M, Larkin J, Lorigan P, Neyns B, Blank CU, Hamid O, Mateus C, Shapira-Frommer R, Kosh M, Zhou H, Ibrahim N, Ebbinghaus S, Ribas A and KEYNOTE-006 investigators: Pembrolizumab versus ipilimumab in advanced melanoma. N Engl J Med 372(26): 2521-2532, 2015. PMID: 25891173. DOI: 10.1056/NEJMoa 1503093

52 Ascierto PA, Del Vecchio M, Mandalá M, Gogas H, Arance AM, Dalle S, Cowey CL, Schenker M, Grob JJ, Chiarion-Sileni V, Márquez-Rodas I, Butler MO, Maio M, Middleton MR, de la Cruz-Merino L, Arenberger P, Atkinson V, Hill A, Fecher LA, Millward M, Khushalani NI, Queirolo P, Lobo M, de Pril V, Loffredo J, Larkin J and Weber J: Adjuvant nivolumab versus ipilimumab in resected stage IIIB-C and stage IV melanoma (CheckMate 238): 4-year results from a multicentre, doubleblind, randomised, controlled, phase 3 trial. Lancet Oncol 21(11): 1465-1477, 2020. PMID: 32961119. DOI: 10.1016/ S1470-2045(20)30494-0

53 Tan PS, Aguiar P Jr, Haaland B and Lopes G: Comparative effectiveness of immune-checkpoint inhibitors for previously treated advanced non-small cell lung cancer - A systematic review and network meta-analysis of 3024 participants. Lung Cancer 115: 84-88, 2018. PMID: 29290267. DOI: 10.1016/ j.lungcan.2017.11.017

54 Moser JC, Wei G, Colonna SV, Grossmann KF, Patel S and Hyngstrom JR: Comparative-effectiveness of pembrolizumab vs. nivolumab for patients with metastatic melanoma. Acta Oncol 59(4): 434-437, 2020. PMID: 31920132. DOI: 10.1080/0284 186X.2020.1712473

55 Prasad V and Kaestner V: Nivolumab and pembrolizumab: Monoclonal antibodies against programmed cell death-1 (PD-1) that are interchangeable. Semin Oncol 44(2): 132-135, 2017. PMID: 28923211. DOI: 10.1053/j.seminoncol.2017.06.007
56 Maxwell R, Luksik AS, Garzon-Muvdi T, Hung AL, Kim ES, Wu A, Xia Y, Belcaid Z, Gorelick N, Choi J, Theodros D, Jackson CM, Mathios D, Ye X, Tran PT, Redmond KJ, Brem H, Pardoll DM, Kleinberg LR and Lim M: Contrasting impact of corticosteroids on anti-PD-1 immunotherapy efficacy for tumor histologies located within or outside the central nervous system. Oncoimmunology 7(12): e1500108, 2018. PMID: 30524891. DOI: $10.1080 / 2162402 X .2018 .1500108$

57 Xing K, Gu B, Zhang P and Wu X: Dexamethasone enhances programmed cell death 1 (PD-1) expression during $\mathrm{T}$ cell activation: an insight into the optimum application of glucocorticoids in anti-cancer therapy. BMC Immunol 16: 39, 2015. PMID: 26112261. DOI: 10.1186/s12865-015-0103-2

58 Jessurun CAC, Hulsbergen AFC, de Wit AE, Tewarie IA, Snijders TJ, Verhoeff JJC, Phillips JG, Reardon DA, Mekary RA and Broekman MLD: The combined use of steroids and immune checkpoint inhibitors in brain metastasis patients: a systematic review and meta-analysis. Neuro Oncol 23(8): 1261-1272, 2021. PMID: 33631792. DOI: 10.1093/neuonc/noab046

59 Petrelli F, Signorelli D, Ghidini M, Ghidini A, Pizzutilo EG, Ruggieri L, Cabiddu M, Borgonovo K, Dognini G, Brighenti M, De Toma A, Rijavec E, Garassino MC, Grossi F and Tomasello G: Association of steroids use with survival in patients treated with immune checkpoint inhibitors: a systematic review and meta-analysis. Cancers (Basel) 12(3): 546, 2020. PMID: 32120803. DOI: $10.3390 /$ cancers 12030546

60 Martins F, Sofiya L, Sykiotis GP, Lamine F, Maillard M, Fraga M, Shabafrouz K, Ribi C, Cairoli A, Guex-Crosier Y, Kuntzer T, Michielin O, Peters S, Coukos G, Spertini F, Thompson JA and Obeid M: Adverse effects of immune-checkpoint inhibitors: epidemiology, management and surveillance. Nat Rev Clin Oncol 16(9): 563-580, 2019. PMID: 31092901. DOI: 10.1038/ s41571-019-0218-0

Received August 21, 2021

Revised September 3, 2021 Accepted September 6, 2021 